\title{
Molecular Orientation Affects Localization Accuracy in Superresolution Far-Field Fluorescence Microscopy
}

\author{
Johann Engelhardt, ${ }^{*}{ }^{\dagger}$ Jan Keller, ${ }^{\ddagger}$ Patrick Hoyer, ${ }^{\dagger}$ Matthias Reuss, ${ }^{\dagger}$ Thorsten Staudt, $^{\dagger}$ and \\ Stefan W. Hell*, ${ }^{*}$,
}

${ }^{\dagger}$ German Cancer Research Center, Im Neuenheimer Feld 280, 69120 Heidelberg, Germany, and

${ }^{*}$ Max-Planck-Institute for Biophysical Chemistry, Am Fassberg 11, 37077 Göttingen Germany

\begin{abstract}
We investigate the cooperative effect of molecular tilt and defocus on fluorophore localization by centroid calculation in far-field superresolution microscopy based on stochastic single molecule switching. If tilt angle and defocus are unknown, the localization contains systematic errors up to about $\pm 125 \mathrm{~nm}$. When imaging rotation-impaired fluorophores of unknown random orientation, the average localization accuracy in three-dimensional samples is typically limited to about $\pm 32 \mathrm{~nm}$, restricting the attainable resolution accordingly.
\end{abstract}

KEYWORDS Microscopy, fluorescence, superresolution, localization, resolution, single molecules

1 he currently most prevalent far-field fluorescence nanoscopy (superresolution) methods overcome the diffraction barrier by employing a molecular mechanism that precludes fluorescence emission when the fluorophores are exposed to excitation light. ${ }^{1}$ This mechanism is utilized so that features closer than the diffraction barrier become fluorescent sequentially in time. Whereas in stimulated emission depletion (STED), ${ }^{2}$ ground-state depletion $(G S D),{ }^{3}$ saturated structured illumination (SSIM), ${ }^{4,5}$ and related methods the fluorescence ability is prepared at a predefined location, ${ }^{1}$ in stochastic optical reconstruction microscopy (STORM), ${ }^{6}$ (fluorescence) photoactivation localization microscopy ((F)PALM), ${ }^{7,8}$ ground-state depletion with individual molecule return microscopy (GSDIM), ${ }^{9}$ or direct STORM (dSTORM) ${ }^{10,11}$ the fluorescence ability of only a single molecule within a region smaller than about half the wavelength of light is released stochastically in space. Since in the latter case the molecular position is unknown, the fluorescence of these individual molecules is projected onto a camera from which the lateral molecular coordinate is determined by centroid calculation, i.e. by localization. ${ }^{12}$

Clearly, the reliability of the images consisting of individual molecular positions critically depends on the precision and accuracy of the localization, making it important to investigate all parameters influencing this procedure. ${ }^{13}$ Notably, it is well-known that the dipole characteristics of fluorophores generally yield asymmetric focal excitation and fluorescence patterns, which are often (somewhat inaccurately) referred to as point-spread functions (PSFs). While

* To whom correspondence should be addressed. E-mail: shell@gwdg.de (SWH); j.engelhardt@dkfz.de (J.E.).

Received for review: 10/2/2010

Published on Web: 12/06/2010 this asymmetry is usually negligible in diffraction-limited imaging, we now show that it can seriously limit the accuracy of the superresolution methods employing single molecule localization.

High-precision localization has been employed to estimate the step sizes of myosin motor proteins, ${ }^{14,15}$ whereby it has been shown that nanometer precision can only be achieved when molecular orientation is taken into account. Furthermore, Bartko and Dickson ${ }^{16}$ and Aguet et al. ${ }^{17}$ demonstrated that the perceived molecular position in total internal reflection fluorescence (TIRF) microscopy depends on molecular orientation, leading to errors as large as \pm 50 $\mathrm{nm}$. In these studies, the molecules of interest were close to the glass/water interface, and also defocused by a known amount to measure the molecular orientation and position. Mortensen et al. ${ }^{18}$ localized single molecules in a TIRF microscope and accounted for asymmetries in the intensity patterns generated by single molecules at the glass-water interface located in the focal plane.

However, if TIRF conditions are now abandoned, which is actually required for a method to be truly far-field, the position of the fluorescent molecules along the optical axis $(z)$ is largely unknown within the range of the axial full width at half-maximum (fwhm). Here we show that, in this case, a systematic error comes into play when the transition dipole of the fluorophore is tilted by an angle $\beta$ with respect to the focal plane $(z=0)$, and the fluorophore is at the same time not in focus $(z \neq 0$ ) (Figure 1$)$. While the precision of the centroid determination still scales inversely with the square root of the number of detected photons, ${ }^{12}$ the location of the centroid strongly depends on the position of the fluorophore along the $z$-axis. 


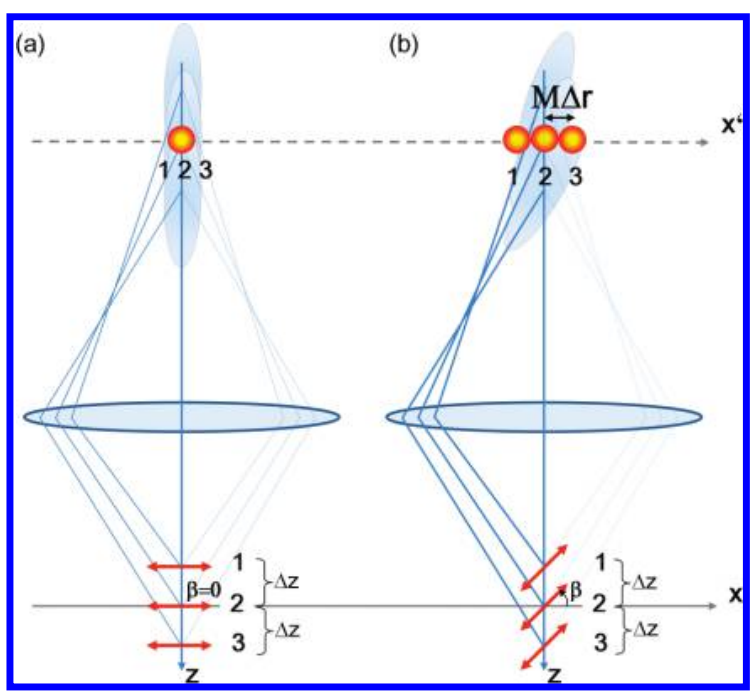

FIGURE 1. A fluorescent molecule whose transition dipole is parallel to the focal plane $(x y)$ appears at the very same lateral position in the image plane irrespective of its position along the optic axis $(z)$ (a). Molecules that are tilted by an angle $\beta$ with respect to the focal plane illuminate the aperture of the objective lens asymmetrically (b). As a result, the PSF is tilted with respect to the optical axis. If the molecule is also defocused, it appears shifted in the image by $\Delta r$ (times the magnification $M$ of the optical system). The apparent location is no longer the actual molecular position.

To investigate the effect, the PSFs rendered by single molecules in a high-aperture microscope were numerically calculated using a vectorial theory. ${ }^{19}$ In epifluorescence imaging, the illumination can be assumed homogeneous over the object plane. The molecular orientations were taken into account by projecting the electric field vectors $\mathbf{E}$ of the excitation and detection field onto the molecular dipole $\mathbf{p}$. The resulting field was squared to obtain the effective intensity distribution of the spot image. In typical (F)PALM, STORM, and GSDIM recordings, the excitation light is focused with low angle, in which case the electrical field vector has a negligible $z$-component.

Calculations were performed for 1.4 numerical aperture (NA) oil immersion lenses with oil refractive index $n=1.518$ and assumed excitation and emission wavelengths of 532 and $600 \mathrm{~nm}$, respectively. A molecule is most efficiently excited and detected if its transition dipole is parallel to the focal plane. Spots with peak intensities $<10 \%$ of the brightest spot (found for $z=0, \beta=0$ ) were omitted, because this threshold level is commonly used in practice. Displacement along the $z$-axis (defocusing) dims the peak brightness of the molecule; at $z \approx \pm 300 \mathrm{~nm}$, it appears half as bright as that at $z=0$. Likewise, a molecule at $z=0$ becomes dimer the more it is tilted out of the focal plane (Figure 2 ). For example, at $\beta \approx 33^{\circ}$, the peak intensity is half of that of the untilted dipole $\left(\beta \approx 0^{\circ}\right)$.

Importantly, defocused, tilted dipoles yield asymmetric diffraction spots having centroids that are shifted from the actual molecular position by $\Delta r$, depending on $\beta$ and $z$. The apparent shift is zero at $\beta=0^{\circ}$ and $90^{\circ}$, reaching its maximum at $\beta \approx 52^{\circ}$. Figure 3 displays the shift for $\beta=$ $45^{\circ}$. The localization precision still scales inversely with the number of detected photons, as expected. However, for molecules randomly oriented in space, the average localization is inaccurate by $\Delta r=32 \mathrm{~nm}$ in the direction of the molecular tilt.

The left-hand side of Figure 3 shows $x y$-images corresponding to slightly different $z$-positions. Note that the spots appear markedly shifted with respect to the real molecular position. At a rather moderate defocus of $z= \pm 200 \mathrm{~nm}$ (see focus series in Figure 3, top left), where the tilted molecule's brightness amounts to $20 \%$ of its maximum (at $z=0, \beta=$ 0 ), the localization error $\Delta r$ is $\pm 65 \mathrm{~nm}$. In other words, if the molecule is located in the range from $z=-200 \mathrm{~nm}$ to $Z$ $=200 \mathrm{~nm}$, the error spans over $130 \mathrm{~nm}$.

The mislocalization is easily recognized in an $x Z$-view of the tilted fluorescence spot (PSF) in the center panel of Figure 3 , showing the optical axis $(z)$ along with the position of the centroids (tilted black line) of their $x y$-sections (PSF in the $x y$-direction) with increasing defocus $z$. For a small defocus of $z= \pm 100 \mathrm{~nm}$, where the molecular brightness amounts to $94 \%$ of its value at $z=0$ and $\beta=45^{\circ}$, the error $\Delta r$ is $\pm 33 \mathrm{~nm}$.

Figure 3 b also shows a calculated subdiffraction "effective PSF" in STED microscopy rendered by a doughnut-shaped STED beam (wavelength $647 \mathrm{~nm}$ ), again from a molecule tilted by $45^{\circ}$. The doughnut is produced by a helical phase ramp ranging from 0 to $2 \pi$, illuminated with circularly polarized light; otherwise the same conditions apply as in the wide field case. Interestingly, the shape and location of the effective PSF rendered by STED is essentially not affected by the severe molecular tilt. If the centroid is calculated from a range of the effective PSF amounting to 3 times its fwhm, the lateral shift is $<2 \mathrm{~nm}$.

Thus it becomes clear that, unless the illumination of the sample or the sample itself is artificially restricted to ultrathin slices, which means abandoning a genuine far-field optical setting, or the fluorophore is tumbling within the fluorescence "on" time, in the single molecule switching methods the accuracy with which the molecular coordinate is established strongly depends on the molecular tilt. For molecules covering the relevant orientation space by rotation within the fluorescence "on" time, the effect of tilt and defocus is averaged out, rendering a spot that is just slightly larger. In this case, the position is still accurate, but the precision is reduced, which can be compensated for by a larger number of detected photons. If the emitting molecules cannot cover the full orientation space around the $z$-axis with nearly equal probability, the localization error needs to be observed. Of high practical relevance is the situation in which tilt and z-position are fully unknown. In this case, an average localization error of $\Delta r=32 \mathrm{~nm}$ limits the realizable resolution to $30-60 \mathrm{~nm}$. The error of the position of individual molecules can be as large as $\Delta r> \pm 100 \mathrm{~nm}$.

In many samples it is not a priori clear whether the tumbling of the fluorophore is free enough. If it is, the 


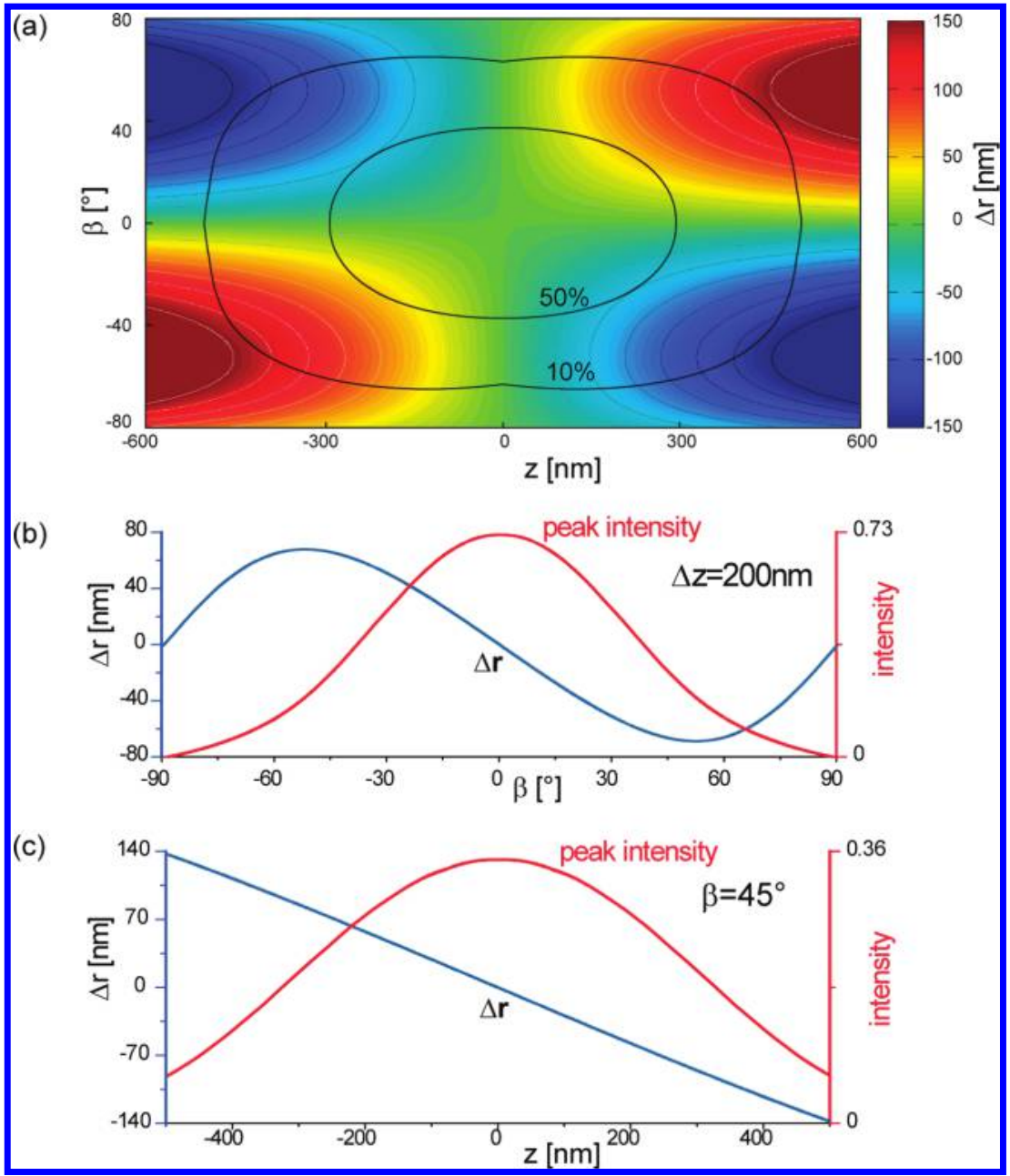

FIGURE 2. The systematic error $\Delta r$ of molecular position gained by centroid calculation depends on the tilt angle $\beta$ with respect to the focal plane $(x, y)$ and on the axial coordinate $(z)$. (a) Dependence of $\Delta r$ on $z$ and $\beta$. Black contour lines depict areas where the molecular brightness is above $10 \%$ or $50 \%$ of the maximum brightness of the molecule found at $z=0$ and $\beta=0$. (b) $\Delta r$ as function of $\beta$ at $z=200 \mathrm{~nm}$ and (c) $\Delta r$ as function of axial position $z$ at $\beta=45^{\circ}$. Defocus and tilt reduce the peak intensity of the diffraction spot (red curve).

question arises whether the orientation cone is covered uniformly. Testa et al., ${ }^{20}$ in their samples containing Mowiol, as well as Gould et al. ${ }^{21}$ performed anisotropy measurements in serialized recordings of switchable emitters showing that a fraction of molecules is fixed in orientation.

To exemplify the shift in molecular position experimentally, we imaged and localized single perylene molecules multiple times at various z-positions. Perylene was selected because of its extreme photostability. The molecules were immobilized in a thin $(\sim 400 \mathrm{~nm})$ spincoated polystyrene layer on a microscope coverslip. To minimize drift effects, the sample was mounted directly at the front end of the objective lens. Figure $4 \mathrm{a}$ shows the superposition of individual molecular positions of a defocus series covering the range between $z=-300 \mathrm{~nm}$ and $z=300 \mathrm{~nm}$. As a drift control, the bottom image shows the superposition of the molecule positions taken before and after the series at $z=$ 0 . The molecules appear at the same locations again, proving that drift was negligible.
Along with the different directions of the localization spread in the top image, Figure $4 \mathrm{~b}$ vindicates that the localization spread observed in Figure $4 \mathrm{a}$ is not due to drift, but is inherent to the recording. Molecules forming essentially circular localization distributions can be attributed to a small tilt angle, whereas pronounced line shaped distributions indicate a large $\beta$. Here the positional uncertainty amounts up to $\Delta r= \pm 100 \mathrm{~nm}$. Thus, the experiment confirms that, when molecules cannot rotate adequately, the centroids of their spots do not yield their coordinate accurately.

Since the inclination of the dipole to the focal plane and the $z$-position are rarely known for labels attached to a threedimensional biological structure, a solution is to use lowviscosity embedding media that facilitate random rotation. TIRF microscopy is less affected because the axial range is restricted to $\sim 150 \mathrm{~nm}$, although the emission field at the optical interface must also be considered. ${ }^{22}$ If multiple focal layers are used to determine $z$-positions of a molecule, ${ }^{23}$ 


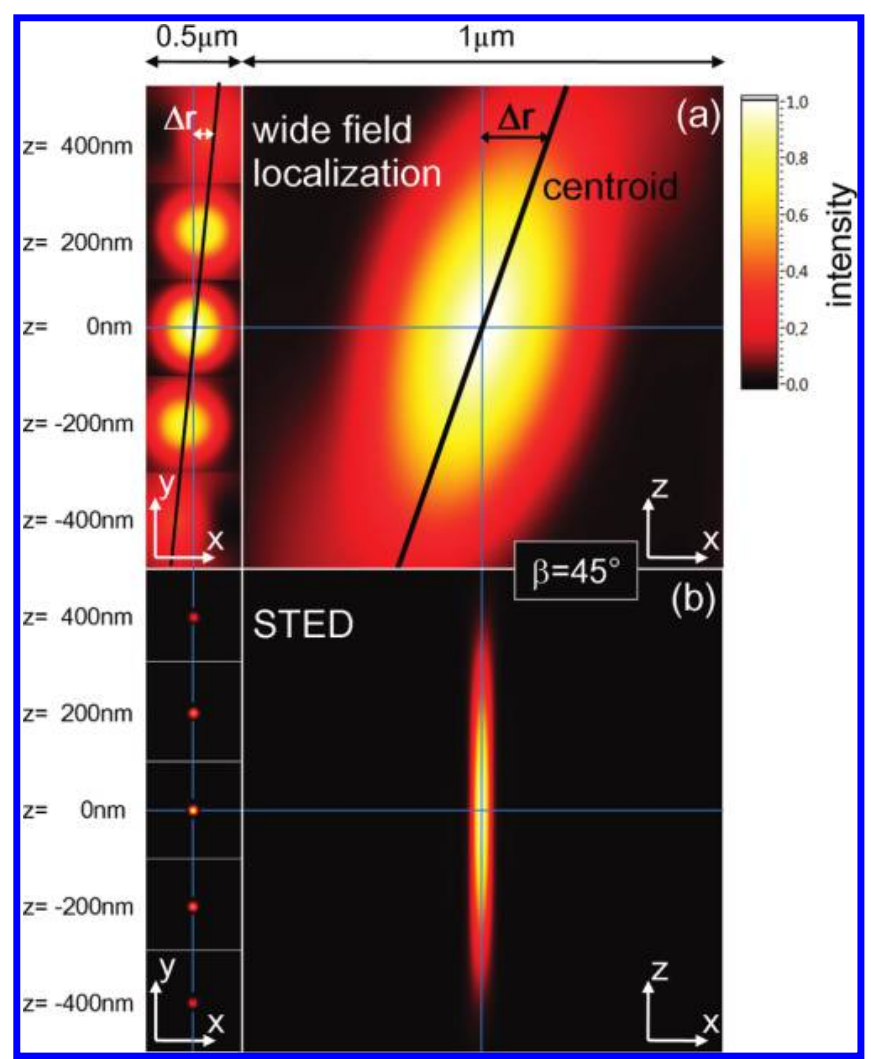

FIGURE 3. Calculated spots (PSFs) produced by a molecule tilted by $\beta=45^{\circ}$ with respect to the focal plane in the $x$-direction, as a function of the coordinate along the $z$-axis, in the image plane of a widefield epifluorescence microscope (a) and in a STED microscope using a vortex doughnut (b). Above, left: $x y$-sections of the diffraction spot at different $z$ coordinates. The lateral deviation of the centers of the spots from the optical axis (central vertical lines) reveals the mislocalization $\Delta r$ of the molecules due to tilt $\beta$ along the $x$-axis. Above right: $x z$-section of the PSF. The black line indicates the position of the centroids depending on the $z$-position. (b) Left: $x y$-spots (PSF) rendered by a STED microscope with lateral 10 -fold resolution increase above the diffraction barrier, here $25 \mathrm{~nm}$, as a function of the $z$-coordinate; the effective PSF is produced by a circularly polarized doughnut-shaped STED beam on the same molecule. Note that, unlike in the epifluorescence detection scheme in STED microscopy, the effect of the molecular tilt is negligible $(<2$ $n m$ ) for all $z$-coordinates.

then fixed tilted molecules are likely to appear at different $x y$-positions. The signal obtained from a single molecule may even be misinterpreted as being two molecules at different positions. Since the spots from an oriented molecule appear slightly elliptical, molecular orientation also needs to be observed when localizing the z-position of molecules using astigmatic beams. ${ }^{24}$ However, the highest care has to be exerted for colocalization measurements, especially when the combinations of defocus and tilt are unfavorable. Since we have exemplified these effects with oil immersion lenses, one should note that in the case of water immersion lenses, additional effects come into play as a result of the glass-water interface.

An alternative to ensuring free rotation is to determine the PSF in three dimensions by shifting the image plane, which is viable when sufficient photons can be gained from

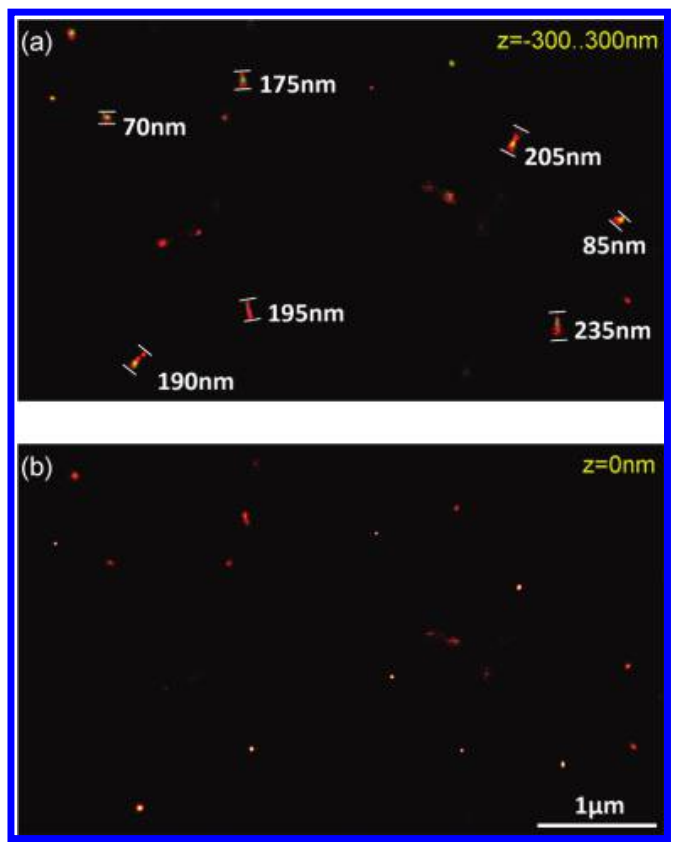

FIGURE 4. Localization error of spatially fixed fluorophores. Depending on defocus $z$ and molecular tilt, single perylene molecules appear at different $x y$-positions following localization by centroid calculation. Images were first acquired in the focal plane $(z=0)$ and then in a series of seven layers with an interlayer distance of $\Delta z=$ $100 \mathrm{~nm}$, corresponding to a total focal range of $\pm 300 \mathrm{~nm}$. (a) Image shows all localized positions of individual molecules taken in the whole series, irrespective of $z$ (i.e., an extended position image). (b) Image displays the positions of the in-focus images $(z=0)$ before and after the series, showing that no notable drift occurred during the $\mathrm{z}$-focus series. In panel a, many of the molecules appear at various positions. Depending on their orientation and focal distance, the positions established for individual molecules are erroneously shifted up to $>200 \mathrm{~nm}$ into different directions as predicted for molecules tilted by $50^{\circ}$ at $z= \pm 300 \mathrm{~nm}$.

a molecule. By considering the nearly linear relationship between defocus and shift (Figure 2), measuring the PSF displacements at different $z$-levels jointly with the corresponding intensity variations is an effective solution for retrieving the correct molecular coordinate by localization.

If the number of detected photons is low, say $<100$, to reliably achieve localization accuracies of $<50 \mathrm{~nm}$, localization microscopy schemes in essence require rotating fluorophores. While they must be attached to the target (bio)molecule rigidly enough to avoid resolution degradation by motion, the fluorophores must also be tumbling. In fact, the incomplete filling of the orientation cone could be one of the reasons why, in many applications, images display a lower actual resolution (i.e., separation capability) than what can be anticipated from the plain number of photons detected from a label. While the tumbling linker may not allow the coverage of the full orientational space, the precision inaccuracy may be alleviated to values significantly smaller than $50 \mathrm{~nm}$. Another remedy is to resort to exceptional fluorophores with a nearly isotropic excitation and emission such as NV centers in nanodiamonds, ${ }^{25}$ or quantum dots. ${ }^{26}$ Note that all calculations implied an infinite number of photons. Clearly, the limited number of emitted photons and the 
emission statistics limits the precision (not the accuracy) of the localization.

The fundamental difference between the nanoscopy family comprising STED, SSIM, and reversible saturable optical fluorescence transitions (RESOLFT) and the PALM, STORM family is that, in the first, the position of the molecules is not found out by localization but predetermined by a light distribution featuring an intensity minimum (zero), i.e., targeted versus stochastic read-out of the emitter coordinate. ${ }^{1}$ In STED and related concepts, the orientation of the fluorophore also matters; ${ }^{27}$ the fluorophores are kept dark by STED only if the electric field of the STED beam is sufficiently strong along the direction of the transition dipole. ${ }^{27-29}$ However, the effect of the dipole orientation and defocus on the position of the STED-PSF peak amounts only to a few nanometers. This is largely because the PSF peak is determined by the predefined position of the zero rendered by the vortex. Only the residual background stemming from fluorescence events that have not been precluded by the doughnut appear shifted. These findings are readily extended to GSD microscopy and to the RESOLFT concept relying on photoswitchable compounds.

In conclusion, unless the molecules are able to rotate relatively freely in space or appropriate remedies are implemented, in stochastic superresolution microscopy approaches using localization, the control of the dipolar orientation is mandatory for attaining spatial resolution and colocalization better than $50 \mathrm{~nm}$.

Acknowledgment. We thank Andreas Schönle and Brian Rankin for fruitful discussions and critical reading. This work was supported by a Gottfried-Wilhelm-Leibniz research award of the Deutsche Forschungsgemeinschaft (to S.W.H.).

\section{REFERENCES AND NOTES}

(1) Hell, S. W. Science 2007, 316 (5828), 1153-1158.

(2) Hell, S. W.; Wichmann, J. Opt. Lett. 1994, 19 (11), 780-782.

(3) Hell, S. W.; Kroug, M. Appl. Phvs. B: Lasers Opt. 1995, 60, 495497.

(4) Heintzmann, R.; Jovin, T. M.; Cremer, C. Opt. Soc. Am. I. A: Opt.. Image Sci., Vision 2002, 19 (8), 1599-1609.

(5) Gustafsson, M. G. L. Proc. Natl. Acad. Sci. U.S.A. 2005, 102 (37), $13081-13086$.
(6) Rust, M. J.; Bates, M.; Zhuang, X. W. Nat. Methods 2006, 3, 793795.

(7) Betzig, E.; Patterson, G. H.; Sougrat, R.; Lindwasser, O. W.; Olenych, S.; Bonifacino, J. S.; Davidson, M. W.; LippincottSchwartz, J.; Hess, H. F. Science 2006, 313 (5793), 1642-1645.

(8) Hess, S. T.; Girirajan, T. P. K.; Mason, M. D. Biophys. I. 2006, 91 (11), 4258-4272.

(9) Fölling, J.; Bossi, M.; Bock, H.; Medda, R.; Wurm, C. A.; Hein, B.; Jakobs, S.; Eggeling, C.; Hell, S. W. Nat. Methods 2008, 5, 943 945.

(10) Bock, H.; Geisler, C.; Wurm, C. A.; Von Middendorff, C.; Jakobs, S.; Schonle, A.; Egner, A.; Hell, S. W.; Eggeling, C. Appl. Phys. B: Lasers Opt. 2007, 88 (8), 161-165.

(11) Heilemann, M.; van de Linde, S.; Schuttpelz, M.; Kasper, R.; Seefeldt, B.; Mukherjee, A.; Tinnefeld, P.; Sauer, M. Angew. Chem. Int. Ed. 2008, 47, 6172-6176.

(12) Heisenberg, W. The Physical Principles of the Quantum Theory; Chicago University Press: Chicago, 1930.

(13) Bobroff, N. Rev. Sci. Instrum. 1986, 57 (6), 1152-1157.

(14) Patra, D.; Gregor, I.; Enderlein, J.I. Phys. Chem. A 2004, 108 (33), 6836-6841.

(15) Toprak, E.; Enderlein, J.; Syed, S.; McKinney, S. A.; Petschek, R. G.; Ha, T.; Goldman, Y. E.; Selvin, P. R. Proc. Natl. Acad. Sci. U.S.A. 2006, 103 (17), 6495-6499.

(16) Bartko, A. P.; Dickson, R. M. L.Phys. Chem. B 1999, 103, 11237 11241.

(17) Aguet, F.; Geissbuhler, S.; Marki, I.; Lasser, T.; Unser, M. Opt. Express 2009, 17 (8), 6829-6848.

(18) Mortensen, K. I.; Churchman, S. L.; Spudich, J. A.; Flyvbjerg, H. Nat. Methods 2010, 7 (5), 377-381.

(19) Richards, B.; Wolf, E. Proc. R. Soc. (London) A 1959, 253, $358-$ 379.

(20) Testa, I.; Schonle, A.; Middendorff, C. V.; Geisler, C.; Medda, R.; Wurm, C. A.; Stiel, A. C.; Jakobs, S.; Bossi, M.; Eggeling, C.; Hell, S. W.; Egner, A. Opt. Express 2008, 16 (25), 21093-21104.

(21) Gould, T. J.; Gunewardene, M. S.; Gudheti, M. V.; Verkhusha, V. V.; Yin, S. R.; Gosse, J. A.; Hess, S. T. Nat. Methods 2008, 5 (12), 1027-1030.

(22) Enderlein, J.; Toprak, E.; Selvin, P. R. Opt. Express 2006, 14 (18), $8111-8120$.

(23) Juette, M. F.; Gould, T. J.; Lessard, M. D.; Mlodzianoski, M. J.; Nagpure, B. S.; Bennett, B. T.; Hess, S. T.; Bewersdorf, J. Nat. Methods 2008, 5 (6), 527-529.

(24) Huang, B.; Wang, W. Q.; Bates, M.; Zhuang, X. W. Science 2008, 319, 810-813.

(25) Jelezko, F.; Wrachtrup, J. Phys. Status Solidi A 2006, 203, 3207 3225.

(26) Bruchez, M.; Moronne, M.; Gin, P.; Weiss, S.; Alivisatos, A. P. Science 1998, 281, 2013-2016.

(27) Dyba, M.; Keller, J.; Hell, S. W. New I. Phys. 2005, 7, 134.

(28) Dedecker, P.; Muls, B.; Hofkens, J.; Enderlein, J.; Hotta, J. I. Opt. Express 2007, 15 (6), 3372-3383.

(29) Reuss, M.; Engelhardt, J.; Hell, S. Opt. Express 2010, 18 (2), 1049 1058. 